Relations industrielles

Industrial Relations

\title{
Le Régime de Rentes du Québec
}

\section{Thaddé Poznansky}

Volume 22, numéro 3, 1967

URI : https://id.erudit.org/iderudit/027814ar

DOI : https://doi.org/10.7202/027814ar

Aller au sommaire du numéro

Éditeur(s)

Département des relations industrielles de l'Université Laval

ISSN

0034-379X (imprimé)

1703-8138 (numérique)

Découvrir la revue

Citer cet article

Poznansky, T. (1967). Le Régime de Rentes du Québec. Relations industrielles / Industrial Relations, 22(3), 422-436. https://doi.org/10.7202/027814ar

Tous droits réservés (C Département des relations industrielles de l'Université Laval, 1967
Ce document est protégé par la loi sur le droit d'auteur. L'utilisation des services d'Érudit (y compris la reproduction) est assujettie à sa politique d'utilisation que vous pouvez consulter en ligne.

https://apropos.erudit.org/fr/usagers/politique-dutilisation/ 


\section{COMMENTAIRES}

\section{QUELQUES COMMENTAIRES SUR LE RÉGIME DE RENTES DU QUÉBEC}

\section{Thaddée Poznanski}

Dans les études qui suivent, nous essayerons de présenter des commentaires sur quelques dispositions du Régime de Rentes du Québec, sans toucher toutefois d̀ la phraséologie et la structure de la loi elle-même, qui pourraient être, à notre avis, sujettes à d'autres commentaires, car elles laissent parfois d désirer. Voici quelques exemples:

1. A lart. 178, au sujet de la rente de veuve, (dans la section régissant les «paiement et conditions des prestations»), il est dit que cla rente de veuve est payable d une bénéficiaire sa vie durant et CESSE A LA FIN DU MOIS DE DECES * et il faut retourner d lart. 123 (dans la section régissant "l'admissibilité aux rentes et prestations *) pour apprendre que "lorsque le bénéficiaire d'une rente de veuve se remarie, cette rente cesse d'être versée à compter du mois qui suit celui du remariage.

Les dispositions semblables s'appliquent à la rente de veuf invalide (art. 179 et 123).

2. A lart. 167, il est écrit "AUCUNE RENTE DE RETRAITE N'EST PAYABLE d un bénéficiaire pour un mois au cours duquel, n'ayant pas atteint 70 ans, il accomplit un travail régulier ». Tandis qu’à lart. 170, on stipule que "la rente de retraite PAYABLE d un bénéficiaire de moins de 70 ans qui accomplit un travail régulier EST REDUIT de la manière prescrite...

3. A lart. 165, il est stipulé que "la rente de retraite est payable à compter du dernier des mois suivants... SANS AUCUNE MENTION concernant les restrictions durant la période de transition contenues à lart. 164.

On pourrait citer plusieurs autres imperfections dans le texte de de la loi en question.

Les mots en italique précédemment sont de l'auteur de ces lignes.

Réduction de la rente de retraite lorsque le bénéficiaire accomplit un travail régulier

La loi sur le Régime de rentes du Québec (RRQ) prévoit, tout comme la loi correspondante du Canada, que la rente de retraite est 
payable, en principe, lorsque le travailleur-cotisant atteint l'âge de 65 ans et n'accomplit pas de " travail régulier "; autrement la rente de retraite n'est exigible qu'à l'âge de 70 ans.

Comme "travail régulier , la loi, à l'article 168 , considère tout travail dont les gains au cours d'une année dépassent $\$ 900$ c'est-à-dire $\$ 75$ par mois; (à compter de l'année 1968, ce montant est sujet aux modifications selon "l'indice des gains », tel que défini par la loi).

La rente de retraite, payable à un bénéficiaire de moins de 70 ans qui accomplit un a travail régulier * et dont les gains de cette source dépassent le dit montant de $\$ 900$, est réduite selon l'art. 170 comme suit:

a) 50 cents pour chaque dollar par lequel les gains de son travail pour l'année excèdent 12 fois son gain mensuel exempté, donc un montant de $\$ 900$, plus

b) 50 cents pour chaque dollar par lequel les gains de son travail pour l'année excèdent 20 fois son gain mensuel exempté, donc un montant de $\$ 1,500$.

Autrement dit (et cela est peut-être plus clair), la réduction en question est égale à la moitié des gains entre $\$ 900$ et $\$ 1,500$ par année, plus la totalité de ces gains dépassant $\$ 1,500$ par année.

Ainsi, par exemple, un bénéficiaire d'une rente de retraite du RRQ, n'ayant pas atteint 70 ans et recevant un revenu d'un "travail régulier * de $\$ 1,600$ par année (environ $\$ 30$ par semaine) verra sa rente réduite pour l'année en question de $\$ 400$, à savoir:

a) $\$ 300$ sur la première tranche de $\$ 600$ dépassant $\$ 900$ et

b) $\$ 100$ sur les gains dépassant $\$ 1,500$.

Tout travailleur, salarié ou autonome, qui n'a pas atteint 70 ans et dont les revenus d'un a travail régulier d dépassent $\$ 2,450$ par année sera, en réalité, privé de sa rente, car, dans la situation actuelle le maximum de la rente de retraite ne peut dépasser $\$ 1,250$ par année (et cela pas avant l'année 1976), tandis qu'avec un revenu d'un a travail régulier > de $\$ 2,450$ la réduction est justement de $\$ 1,250$, à savoir: $\$ 300$ sur la moitié de la tranche entre $\$ 900$ et $\$ 1,500$, plus $\$ 950$ c'est-à-dire l'excédent total sur $\$ 1,500$. Dans plusieurs cas, un revenu d'un travail régulier même au-dessous de $\$ 2,450$ va priver le bénéficiaire complètement de sa rente, ceci dans les cas où la rente de retraite, calculée sur la moyenne de ses gains admissibles, n'atteindra pas, même après 1976, $\$ 1,250$ par année, c'est-à-dire $25 \%$ des gains moyens admissibles avec un maximum des gains admissibles de $\$ 5,000$ par année ${ }^{1}$

(1) Remarquons que la mention dans le Bulletin No 1 du RRQ est inexacte, lorsqu'on y stipule que la rente de retraite est égale à $25 \%$ du revenu moyen du cotisant, a le dit revenu moyen ne pouvant toutefois excéder $\$ 5,000 *$, dit le Bulletin. Ce n'est pas le revenu moyen qui ne peut excéder (à l'heure actuelle) $\$ 5,000$, mais le revenu admissible pour chaque année; cela n'a pas le même effet. 
Dans le même Bulletin, on a confondu a l'âge d'admission à la rente de retraite , avec celui de retraite elle-méme, en disant que a l'âge de la retraite sera abaissé graduellement pendant les cinq premières années du Régime * ou que "la retraite est obligatoire à 70 ans $»$.

En réalité, la loi sur le RRQ ne parle nullement de l'áge de la retraite, mais uniquement de l'âge de l'admissibilité à la rente de retraite.

Il peut donc arriver fréquemment qu'aucune rente du RRQ ne sera versée (ou on exigera le remboursement) aussi longtemps que le bénéficiaire n'a pas 70 ans, ceci lorsque la rente elle-même n'atteint pas le montant des réductions prévues par la loi.

Signalons cependant que la rente de retraite n'est jamais réduite pour un mois durant lequel les gains du a travail régulier * du bénéficiaire n'excèdent pas $\$ 75$.

Le principe que le montant de la rente de retraite est subordonné au fait qud le cotisant, salarié ou indépendant, ne fait plus partie du marché du travail, c'est-à-dire qu'il n'accomplit aucun " travail régulier * (au sens de la loi) se retrouve aussi dans plusieurs régimes à l'étranger. Le comité interministériel d'étude sur le Régime de rentes du Québec explique sa recommandation à ce sujet comme suit:

- Un certain nombre de bénéficiaires de rente de retraite feront encore partie de la main-d'oeuvre ou recevront un revenu provenant de leur travail. En effet, d'après le recensement de 1961, la proportion des travailleurs par rapport à la population totale entre 65 et 69 ans inclusivement, dans la province, s'élève à $44 \%$ pour les hommes et à $11 \%$ pour les femmes. Par suite de l'importance de ces pourcentages, le Comité est d'opinion qu'il est essentiel de conditionner la rente de retraite au retrait quasi-total de la main-d'oeuvre, car autrement :

* a) le coût du régime serait inutilement haussé de façon très appréciable ; et,

«b) le cumul de la rente de retraite, de la pension de la sécurité de la vieillesse anticipée * et du revenu provenant du travail aurait pour effet de hausser temporairement le revenu de ces travailleurs et par conséquent de majorer leurs exigences après leur retrait définitif de la main-d'oeuvre. Il en résulterait inévitablement une hausse indirecte du coût de la sécurité sociale.

« Toutefois, malgré ces raisons, le Comité croit que du point de vue social, il est bon de permettre au travailleur à la retraite d'effectuer de menus travaux et de supplémenter, dans des limites raisonnables, son revenu de retraite.

* A l'époque, le projet fédéral prévoyait la possibilité d'une pension de la sécurité de la vieillesse anticipée (T.P.). 
* Puisque le paiement de la sécurité de la vieillesse est accordé, sans condition, à compter du 70ème anniversaire, le Comité est d'opinion qu'à compter de cet âge le paiement de la rente de retraite ne doit plus être conditionné à l'abandon du travail rémunérateur. Un examen des taux de participation de la population à la main-d'oeuvre, selon le recensement de 1961, révèle d'ailleurs qu'environ $90 \%$ des travailleurs ont pris leur retraite avant 70 ans. Une telle formule est très souple puisqu'elle accorde le degré désiré de liberté au participant quant à la date de sa retraite et qu'elle lui permet de retourner au travail occasionnellement ou à temps complet sans que les objectifs du régime soient compromis. .

Le principe de réduction de la rente de retraite (et même d'annulation, pour toute fin pratique de son effet) aux bénéficiaires recevant un revenu provenant du travail avant un certain âge (selon le RRQ et le régime canadien de 70 ans) est fortement critiqué.

D'ailleurs, on ne retrouve pas une telle réduction dans les régimes concernant les fonctionnaires publics, sauf, si le bénéficiaire reçoit à la retraite un revenu de la même source ou d'une source semblable.

Voici la situation, au Québec, des fonctionnaires provinciaux et des enseignants qui, à leur retraite, accomplissent un travail rénumérateur.

La loi québecoise sur le "Régime de retraite des fonctionnaires * prévoit (à l'art. 31) la suspension de la pension de retraite lorsque le bénéficiaire occupe, à titre temporaire ou permanent, une charge, une fonction ou un emploi auquel est attachée une rémunération payée par le gouvernement de la province ou par un office, une commission ou une régie relevant de ce gouvernement; toutefois, lorsque la rémunération annuelle ou calculée sur une base annuelle, est inférieure au montant annuel de la pension, le bénéficiaire continue à recevoir la différence entre la rémunération et le montant de la pension (art. 32).

D'après la loi québecoise sur le Régime de retraite des enseignants est réduite dès que le titulaire occupe une fonction d'enseignant ou d'un fonctionnaire, ou employé provincial et reçoit un traitement excédant $30 \%$ du traitement moyen qui sert de base à la fixation de la pension. $\mathrm{La}$ réduction dans un tel cas, est égale à cet excédent.

Par contre, ni la loi concernant le Régime de retraite des fonctionnaires, ni celle concernant le Régime de retraite des enseignants ne pénalisent un bénéficiaire de la pension de retraite qui occupe une position lucrative dans une entreprise privée ou à son compte; bien entendu, la partie de la pension qui est versée à un tel bénéficiaire par le RRQ est soumise à la réduction comme c'est le cas envers tous les autres bénéficiaires.

Et il faut rappeler ici, que ces pensions sont souvent accordées avant l'áge de 65 ans, par exemple après 35 ans de service, ce qui peut ame- 
ner que la pension de retraite soit payée déjà à partir de l'âge de 55 ans et même à un âge plus jeune.

Revenant à la réduction prévue dans le RRQ et préconisée, comme nous l'avons fait remarquer, dans le rapport du Comité Interministériel, il est à mentionner, en premier lieu, que depuis la préparation de ce rapport (publié en avril 1964) l'âge de la pension de la sécurité de la vieillesse est avancé; dès l'année 1970, elle sera versée sans condition (quant au travail) à compter de l'âge de 65 ans.

D'autre part, la philosophie préconisée qu'il faut dans la mesure du possible, éliminer les pensionnaires du marché du travail, est souvent critiquée. Dans plusieurs pays où de telles restrictions existent, on constate une tendance à les éliminer, cela entre autres, à cause de la raréfaction des ressources humaines et surtout de travailleurs spécialisés.

En Belgique, par exemple, où les bénéficiaires d'une rente de retraite sont autorisés à exercer, dans les limites très strictes, une certaine activité professionnelle, ces limites étaient de 60 heures ou 1,800 francs par mois (env. \$40), il semble qu'on veuille augmenter ces limites à 90 heures et 2,700 francs. Les demandes d'abolir les restrictions viennent aussi bien du patronat que du salariat; ces derniers soulignent e. a. la discrimination entre les retraités du secteur public (très nombreux en Belgique) et ceux du secteur privé.

La même tendance à ne pas faire d'obstacles aux travailleurs âgés mais ayant l'expérience, se rencontre dans quelques pays communistes; malgré l'explosion démographique aussi là-bas, on trouve qu'un travailleur âgé mais expérimenté vaut pour la production plus qu'un travailleur jeune sans expérience.

\section{Sur les gains admissibles de travailleurs autonomes (indépendants)}

D'après le principe de la loi sur le Régime de rentes du Québec (RRQ), tout travail dans la province est visé par ce Régime de sorte que tout travailleur, salarié ou autonome (indépendant) est assujetti aux dispositions du Régime; la même chose, d'ailleurs, s'applique aux autres provinces conformément aux principes du * régime de pensions du Canada ».

Le Comité interministériel d'étude sur le Régime de rentes du Québec a recommandé, dans son rapport, que tous les travailleurs dépendants et indépendants soient tenus d'adhérer au Régime. Parmi les motifs invoqués à ce propos, on peut lire, entre autres, ce qui suit:

* Le régime est une mesure de sécurité sociale. Il est donc important que la plus grande partie possible de la population bénéficie de ses avantages. Il est, cependant, de rigueur d'exclure les personnes qui n'ont point de revenu de travail par suite de la nécessité ; 
* a) que le coût du régime soit supporté uniquement et directement par ses cotisants (travailleurs dépendants et indépendants et leurs employeurs) ;

«b) que le montant des prestations soit relié, en principe, au revenu du cotisant. »

Le rapport ajoute:

"Comme les revenus provenant de sources autres que le travail sont susceptibles de continuer au décès ou à la retraite, le Comité juge, de plus, nécessaire de limiter les revenus aux fins du régime à ceux provenant du travail.

"De plus, selon le Comité, aucune personne ne devrait bénéficier de la redistribution qui favorise certaines catégories de participants par suite de revenus provenant de sources autres que le travail. »

En appuyant sur les principes de l'adhésion obligatoire, le Comité n'as pas manqué de signaler que "il est facile d'imaginer que si l'adhésion était facultative, il $\mathrm{y}$ aurait une concentration de cotisants parmi les catégories qui pourraient retirer les plus grands avantages».

Nous mentionnons ceci pour analyser et commenter la disposition du RRQ concernant les "gains admissibles " provenant du travail autonome.

En ce qui concerne le salaire admissible d'un travailleur a dépendant . (salarié), c'est le revenu qu'il retire en exécutant un contrat de louange de service personnel ou l'exercice d'une charge, revenu calculé selon la Loi de limpôt provincial sur le revenu, plus toutes les déductions prévues par cette loi.

D'autre part, pour les gains du travail autonome, la loi sur le RRQ précise à l'art. 44, que ceux-ci "sont un montant égal à son revenu pour l'année provenant de toutes ses entreprises, autres qu'une entreprise dont plus de $50 \%$ du revenu brut provient de la location d'immeubles. \

Parmi les travailleurs indépendants ou autonomes, il y a ceux dont les revenus proviennent uniquement ou presque exclusivement de leur travail; ce sont, par exemple, les avocats, notaires, médecins, dentistes, comptables, quelques artisans, etc, tandis qu'il y en a d'autres dont les revenus proviennent aussi bien de leur travail personnel que du capital investi dans leurs entreprises; ce sont, entre autres, les fermiers, les commerçants, les petits industriels, etc.

Il est à remarquer que la loi (à l'art. 1) définit le " travail autonome * comme étant un travail qu'un particulier exécute pour son propre compte et que l'expression "entreprise * signifie toute activité lucrative autre 
qu'une charge ou un travail exécuté par un salarié; les revenus d'un tel travail sont considérés comme * salaires *, la loi (à l'art. 1) définissant le * travail » comme étant *l'exécution d'un contrat de louage de service personnel ou l'exercice d'une charge *, et l'expression "charge * comprend . le poste qu'occupe un particulier et qui lui donne droit à une rémunération. .

Dans la présente étude, nous nous occuperons seulement des * travailleurs autonomes », donc des personnes exécutant un travail pour leur propre compte. Comme nous l'avons indiqué précédemment, les gains admissibles pour le RRQ de telles personnes sont égaux à leurs revenus provenant de toutes leurs entreprises (avec une seule exception concernant les revenus provenant de la location d'immeubles); la loi précise expressément que les revenus en question doivent être calculés selon la Loi de l'impôt provincial sur le revenu. D'après cette dernière loi, un travailleur autonome doit déclarer, en plus des revenus éventuels provenant d'un contrat de travail, tous ses autres revenus, sans faire la distinction entre les revenus provenant du capital investi dans son entreprise ou provenant de ses placements et ceux qu'il peut avoir par son travail personnel dans la dite entreprise.

Ceci concerne, entre autres, tous les petits commerçants ou industriels s'occupant personnellement, avec ou sans aide étrangère, de leur commerce ou de leur industrie, de même que tous les cultivateurs (fermiers) travaillant sur leur ferme.

D'après la teneur de la Loi, les cotisants de telles catégories seraient assujettis quant aux gains admissibles au RRQ pour le revenu total, sans distinction de la source et de la provenance, bien entendu dans les limites prescrites par le RRQ. Il nous semble que ceci soit contraire à l'esprit de l'assurance sociale en soumettant au RRQ, non seulement les revenus de telles personnes provenant de leur travail, mais aussi ceux provenant du capital investi et des autres placements, etc, car dans de tels cas, aussi bien les cotisations que les prestations seraient fixées en fonction de leur revenu global. A première vue, cela pourrait paraître que de tels travailleurs autonomes soient obérés, la loi les forçant à payer, à titre de cotisation, un montant trop élevé, en incluant dans le gain admissible le revenu du capital. Mais, en réalité, toutes ces catégories de travailleurs autonomes sont favorisées, car grâce à la distribution des ressources qui est à la base du système financier du Régime, les travailleurs de ces catégories profitent énormément, lorsque le revenu du travail proprement dit, n'atteint pas le maximum des gains cotisables $(\$ 5,000$ par année, à l'heure actuelle). Il ne faut pas perdre de vue que de tels travailleurs autonomes ayant un capital dans leur entreprise et étant, règle générale, d'âge mûr, vont jouir de la rente de retraite (et des autres prestations du RRQ) prochainement ou dans quelques années, de sorte que la valeur des prestations à recevoir dépasseront de beaucoup la valeur de leurs cotisations, même 
s'ils sont obligés de payer seuls (c'est-à-dire sans le concours de leur employeur) toute la cotisation de $3.6 \%$ du revenu cotisable.

Il nous semble donc, que les dispositions du RRQ ne respectent pas les observations faites par le Comité d'Etudes que nous avons cités au début de ces notes. Il est à signaler aussi que dans l'exposé des principes du projet de la loi sur le Régime de rentes du Québec, présenté à la Législature au nom du Gouvernement par le Comité du Régime il est expressément dit que "le revenu considéré est celui qui provient du travail à lexclusion de tout autre. Ainsi, le revenu du placement de capitaux ne sera pas sujet d cotisation..

Il est évident pour nous, que tout travailleur autonome d'âge mûr tirant ses revenus d'une entreprise dans laquelle il a investi un capital ou ayant quelques revenus de placements, etc, a l'avantage de payer la cotisation au RRQ sur son revenu global, y compris celui provenant du capital investi et de ses placements, etc., car les prestations à recevoir dépasseront de beaucoup les cotisations à verser.

Pour prouver que nous ne nous trompons pas dans l'interprétation des dispositions du RRQ concernant les gains admissibles des travailleurs autonomes, nous pouvons citer quelques extraits du Bulletin de la RRQ d'avril 1966, donnant des explications "Pour le cultivateur Comment calculer le revenu net ». Voici ce qu'on peut y lire:

- Pour les fins du Régime de rentes, le cultivateur qui exploite sa propre ferme est considéré comme un travailleur à son compte. Le taux de sa contribution est donc de 3.6 pour cent de son revenu net moins l'exemption générale de $\$ 600$. La contribution est obligatoire lorsque le revenu net dépasse $\$ 800$.

- Le revenu qui sert au calcul de la contribution d'un cultivateur est le revenu net de sa ferme plus le revenu net de toute autre entreprise qu'il exploite. Ce revenu ne comprend pas cependant les revenus de placements tels que dividendes et intérêts, ni le revenu de loyers.

\& Qu'est-ce que le revenu net d'une ferme?

- Le revenu net d'une ferme est égal à la différence entre le revenu brut et les dépenses faites pour l'exploitation de la ferme, comme le montre l'exemple suivant :

- Revenu brut

- Moins dépenses d'exploitation

- Revenu net 
" Comment calculer le revenu net?

« L'exemple ci-dessus nous montre que pour calculer le revenu net d'une ferme, il faut d'abord connaître le montant de son revenu brut et celui des dépenses d'exploitation.

- Le revenu brut d'une ferme peut provenir de plusieurs sources. Il comprend, par exemple, la valeur des produits et des animaux vendus, la valeur des produits agricoles ou des animaux consommés sur la ferme, ainsi que toute ristourne, prime à l'exploitation ou paiement de soutien des prix.

- Les dépenses, pour leur part, sont d'autant plus nombreuses que la production de la ferme est importante et variée. Elles peuvent comprendre, par exemple, les salaires, l'achat d'animaux, les taxes, l'intérêt sur les emprunts, les assurances sur les bâtiments ou la machinerie, l'entretien et la réparation des instruments aratoires, les frais de vétérinaire, etc.

* Pour illustrer plus clairement la façon d'établir le revenu net d'une ferme, voici un exemple donnant une idée des sources de revenu et de dépense les plus fréquentes. Il va sans dire que les chiffres mentionnés sont pris au hasard et ne correspondent pas nécessairement à la réalité.

\section{Revenu brut :}

Ventes de produits: lait, foin, pommes, etc. $\$ 3,350.00$

Vente d'animaux

$2,500.00$

Travaux exécutés à forfait

250.00

Valeur des produits consommés sur la ferme $\quad 650.00$

Divers

$400.00 \quad \$ 7,150.00$

\section{Dépenses d'exploitation:}

Salaires

$\$ 350.00$

Taxes

290.00

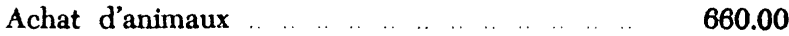

Entretien et réparation de tracteur ........ . 450.00

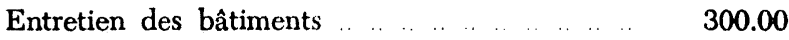

Achat de semences, de moulées ......... . . $\quad 870.00$

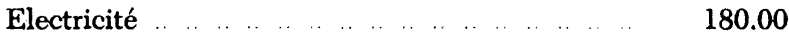

Dépréciation sur machinerie et bâtiments . .. $1,100.00$

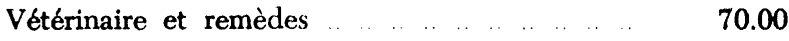

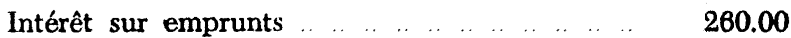

Assurance sur machinerie et bâtiments .. . .. $\quad 90.00 \quad \$ 4,620.00$

Revenu net 
Contribution au Régime de rentes:

Si nous continuons d'utiliser l'exemple ci-dessus, le calcul de la contribution au Régime de rentes s'effectue de la façon suivante :

Revenu net de la ferme

$\$ 2,530.00$

Moins exemption générale 600.00

Revenu cotisable

Taux de contribution

Contribution

$\$ \quad 69.48$

La citation ci-dessus est extraite littéralement du Bulletin de la RRQ. Nous laissons sans commentaires le fait, qu'on y considère la vente ou l'achat des animaux comme "revenus ou dépenses", selon le cas. Est-ce que ces postes ne sont pas plutôt, au point de vue comptable, des * recettes et déboursés * ? Est-ce que l'achat des animaux n'augmente pas parfois la fortune du contribuable sans diminuer le "revenu * (dans les sens comptable de ce mot)?

Il est à noter que parmi les dépenses, on cite «lintérêt sur emprunts ", tandis que l'intérêt sur son capital investi n'y figure pas. Cela provient du fait qu'on assimile les gains admissibles (ou cotisables) pour le RRQ au revenu imposable pour l'impôt sur le revenu qui englobe (et doit englober) le revenu du capital investi de même que les revenus des placements, etc.

Les travailleurs autonomes dont il est question dans cette étude, loin d'être pénalisés par les dispositions du RRQ, au contraire en profitent, parfois même d'une manière substantielle. Peut-on éviter une telle - spéculation - qui est parfaitement légale? Il nous semble que la loi du RRQ devrait s'en tenir strictement aux principes énoncés par le Comité d'Etude et ne considérer comme "gains admissibles " que ceux qui proviennent du travail proprement dit. Dans le rapport du Comité d'Etude, on trouve une mention (volume 1, p. 45) que:

- le Comité a considéré la possibilité d'une cotisation fixe de la part de certaines catégories de travailleurs indépendants qui pourraient hausser fictivement le montant de leur revenu provenant du travail au cours des années qui précèdent la retraite ou pour lesquels travailleurs il pourrait être difficile de déterminer la partie de leur revenu provenant du travail. Cette possibilité a été rejetée par suite de la difficulté de déterminer ces catégories spéciales ainsi que le montant de la cotisation fixe.

Et le rapport ajoute:

- Cependant, la Régie pourra, si nécessaire, fixer à forfait le revenu provenant du travail pour certaines catégories de travailleurs indépendants. . 
Nous sommes fermement d'opinion qu'une telle fixation du revenu forfaitaire s'impose envers les catégories des travailleurs autonomes dont il est question dans ces notes et si la teneur de l'art. 44 de la Loi sur le RRQ ne le permet pas, il faudra, à notre avis, amender la loi, parce que le danger d'* exploiter * légalement la RRQ est grand.

D'ailleurs, il existe d'autres possibilités, pour les travailleurs indépendants, de se servir abusivement, mais légalement, du RRQ. Ainsi, par exemple, la Loi prévoit à l'art. 51, que tout travailleur indépendant, dont les gains provenant du travail sont inférieurs à $\$ 800$ pour l'année ( 1 et $1 / 3$ fois $\$ 600$ ) est exempté de la contribution (ou cotisation) pour le RRQ et, par conséquent les périodes où les gains effectifs n'atteignent pas $\$ 800$ par année, ne seront pas prises en considération pour le calcul des prestations. Existe-t-il un empêchement à l'effet qu'un tel travailleur déclare à l'impôt sur le revenu, un montant dépassant $\$ 800$, par exemple $\$ 2,000$ et plus? Règle générale, il ne payerait pas d'impôt (vu les exemptions personnelles), tandis que la contribution annuelle pour le RRQ serait de $\$ 50.40$ par année pour un revenu de $\$ 2,000$ (3.6\% de $\$ 1,400)$; tandis que la rente de retraite payable après des gains annuels de $\$ 2,000$ après 10 ans, serait de $\$ 500$ par année tandis que le total des contributions pendant 10 ans serait $\$ 504$ dont la moitié, d'après la nouvelle disposition légale, sera remboursée au contribuable par le fisc. Ainsi un profit très substantiel. Comment prévenir de tels abus?

Dans tout régime de rentes dont le système financier est basé sur la répartition des ressources entre les différentes classes ou groupes d'assurés (quant aux générations, salaires, etc) et non sur l'équivalence individuelle entre les contributions et les prestations de chaque participant, il est toujours possible qu'on trouve quelques imperfections ou « anomalies » et que les personnes adéquatement renseignées à ce sujet en profitent a légalement *. Les lois en question ne devraient donc pas encourager aussi facilement ces genres d'abus.

\section{Le « trop perçu * de la part des employeurs}

Dans toute assurance sociale, les contributions (cotisations) qui incombent aux salariés sont - règle générale - déduites par l'employeur à chaque paie (rémunération) versée à son travailleur et remises à lautorité qui administre l'assurance en question; c'est ce qu'on désigne "déduction à la source ».

Le RRQ, tout comme le Régime de pensions du Canada (RPC), a adopté le même principe (voir l'art. 56 du RRQ et l'art. 22 du RPC). D'autre part, dans ces systèmes, l'employeur doit payer une contribution égale à celle déduite du salaire de l'employé aux fins du Régime.

Les déductions à la source se font, en principe, selon une formule qui tient implicitement compte du fait que le travailleur en question aura le même revenu (salaire) durant toute l'année. Ainsi, par exemple, 
la contribution du travailleur, recevant un salaire mensuel de $\$ 300$, est de 4.50 par mois calculée de la façon suivante: la rémunération mensuelle de $\$ 300$ est réduite de $1 / 12$ de l'exemption personnelle générale $(\$ 600)$, il reste donc $\$ 250$ sur lequel montant la contribution de $1.8 \%$ est de $\$ 4.50$. Mais si, pour une raison quelconque, ce salarié ne travaille que durant 10 mois, les déductions pour l'année s'élèveraient à $\$ 45.00$, tandis qu'avec un revenu annuel de $\$ 3,000$ (10 fois $\$ 300$ ), sa contribution ne devrait être que de $\$ 43.20$, c'est-à-dire $1.8 \%$ sur $\$ 2,400$; la différence serait plus grande s'il n'avait travaillé que durant 6 mois, formant un gain annuel de $\$ 1,800$; dans ce cas, la contribution serait de $\$ 21.60$ c'està-dire $1.8 \%$ sur $\$ 1,200$, tandis que la déduction à la source représenterait $\$ 27.00$ (6 fois $\$ 4.50$ ).

Font aussi partie de cette catégorie, les travailleurs marginaux qui travaillent seulement quelques semaines durant l'année et dont les revenus n'atteignent pas $\$ 600$ par année; l'employeur doit néanmoins déduire la contribution qui est calculée dans l'hypothèse que ce travailleur recevra le même salaire toute l'année. Ainsi, par exemple, pour un salaire hebdomadaire de $\$ 50$, la déduction à la source est de $\$ 0.69$; s'il ne travaille que 10 semaines durant l'année civile, il aurait payé $\$ 6.90$ à titre de contribution par la déduction à la source, tandis qu'en réalité avec un revenu de $\$ 500$, il n'est pas admissible, donc les contributions lui seront remboursées.

En effet, la loi sur le RRQ, tout comme celle du RPC, prévoit aux articles 48 et 91 (resp, art. 8 et 39) qu'un travailleur salarié, auquel on a déduit à la source plus que $1.8 \%$ du total de son salaire cotisable, c'est-à-dire de son salaire admissible (limité actuellement à $\$ 5,000$ par année) moins l'exemption personnelle générale de $\$ 600$ par année, a droit au remboursement de l'excédent.

Le phénomène que les déductions à la source sont susceptibles de dépasser le montant légalement admissible provient de causes différentes, dont nous en énumérerons quelques-unes.

L'une de ces causes consiste dans le fait que le taux effectif de contribution du travailleur n'est pas uniforme, car l'exemption générale de $\$ 600$. par année ( $12 \%$ du maximum des salaires admissibles, c'està-dire du ' $\$ 5,000$ ) produit un taux progressif, par exemple il n'est que:

$0.72 \%$ pour un revenu annuel de $\$ 1,000$ (1.8\% de $\$ 400)$

$1.44 \%$ pour un revenu annuel de $\$ 3,000$ (1.8\% de $\$ 2,400)$

$1.50 \%$ pour un revenu annuel de $\$ 3,600$ (1.8\% de $\$ 3,000)$

$1.58 \%$ pour un revenu annuel de $\$ 5,000$ (1.8\% de $\$ 4,400)$

Une autre cause résulte du fait que pour les salariés gagnant plus que le maximum annuel admissible $(\$ 5,000$ à l'heure actuelle) la déduction à la source est calculée de telle façon que le plein montant de la contribution (\$79.20) est déduit avant l'expiration de l'année civile 
en cours. Ainsi, par exemple, pour un salarié recevant $\$ 12,000$ par année, à raison de $\$ 1,000$ par mois, la déduction à la source est de $\$ 17.10$ par mois, selon le calcul suivant: $1.8 \%$ de $\$ 950$, c'est-à-dire $\$ 1,000$ moins $\$ 50$ ou $\$ 50$ est $1 / 12$ de l'exemption générale de $\$ 600$. Bien entendu, le maximum de la contribution pour l'année ne peut excéder $\$ 79.20$ $(1.8 \%$ de $\$ 4,400)$ de sorte que les déductions après cinq mois cessent et même la déduction durant le cinquième mois n'est que $\$ 10.80$ pour compléter à $\$ 79.20$, les quatre déductions précédentes de $\$ 17.10$. Or, si un tel travailleur change d'employeur durant l'année civile, par exemple après 5 ou 6 mois, le nouvel employeur sera obligé de faire de nouvelles déductions sans se préoccuper du fait que notre travailleur a déjà contribué le maximum prévu par la loi. ${ }^{2}$

La troisième cause que nous nous proposons de décrire ici, provient du fait que certains salariés peuvent avoir simultanément ou concurremment plusieurs employeurs (par exemple, régulièrement ou pour les fins de semaines, pendant les vacances, etc). Chaque employeur étant obligé de déduire à la source, la contribution selon le salaire payé, il peut arriver que le total de ces déductions durant l'année dépasse le maximum de $\$ 79.20$.

Remarquons en passant, que le fait d'avoir, simultanément ou consécutivement durant l'année, deux (ou plusieurs) employeurs peut conduire à la réduction du total des contributions d'un tel travailleur, et conséquemment des salaires admissibles pour l'année en question donc des prestations qui, règle générale, sont basées sur ces salaires admissibles. Recevant, par exemple, des revenus durant l'année de \$2,500 de chacun des deux employeurs, donc un total de $\$ 5,000$, la déduction à la source serait dans chaque cas de $\$ 34.20$ durant l'année $(1.8 \%$ de $\$ 1,900$, les premiers $\$ 600$ n'étant pas cotisables) donc un total de $\$ 68.40$ tandis qu'un salaire unique de $\$ 5,000$ exige une contribution de $\$ 79.20$.

Conformément à l'article 53 de la loi québecoise (* Conciliation des données à l'égard du salaire ») ou l'article 14 du RPC, le salaire global d'un tel travailleur sera calculé sur la base du total des déductions à la source durant l'année et cela selon la formule $500 / 9$ du total des déductions, soit $\$ 3,800$ donc ale salaire admissible (pour le calcul des prestations) deviendra pour cette année de $\$ 4,400$, c'est-à-dire $\$ 3,800$ plus $\$ 600$ de l'exemption générale. La différence avec le salaire effectif global de $\$ 5,000$ provient justement du jeu que les taux de contributions ne sont pas uniformes, mais progressifs de sorte que les déductions sur deux salaires à $\$ 2,500$ sont moindres que celles sur $\$ 5,000$.

Cependant, un tel salarié peut choisir l'option prévue à l'article 52 du RRQ (ou au paragraphe 3 de l'article 12 du RPC) et demander

(2) Cela peut arriver, par exemple, assez fréquemment parmi les instituteurs qui changent d'employeur vers le ler juillet. 
avant le 30 avril de l'année suivante qua l'excédent de son salaire admissible (réduit de son exemption personnelle) sur le salaire, calculé selon les déductions à la source (conformément au principe de l'article 53 du RRQ ou larticle 14 du RPC), donc dans notre exemple $\$ 600$, c'est-à-dire la différence entre le montant de $\$ 4,400$ ( $\$ 5,000$ moins $\$ 600$ ) et $\$ 3,800$, soit considéré comme gain admissible du travail autonome, sur lequel gain le travailleur pourra verser la contribution à raison de $3.6 \%$; il est entendu que la limite globale doit être respectée.

Revenant aux causes principales où les contributions déduites à la source peuvent dépasser le montant légalement exigible, le travailleur concerné, comme nous l'avons dit, a droit au remboursement et ce remboursement en vertu du premier paragraphe de l'article 93 du RRQ (ou l'article $39 \mathrm{du}$ RPC) peut se faire même sans une demande de sa part à cette fin.

En vertu de l'article 49 du RRQ (ou l'article 9 du RPC) l'employeur doit, comme nous l'avons mentionné auparavant, pour chacun de ses salariés, verser un montant égal à celui qui est déduit de la rémunération du travailleur. Mais si les lois en question prévoient explicitement le droit au remboursement du trop perçu aux travailleurs-salariés, elles ne font aucune mention, par ailleurs, d'un tel remboursement des excédents aux employeurs. On en déduit, avec raison, que les employeurs n'ont pas droit au remboursement des sommes payées en trop pour les salariés; cette omission n'est pas un oubli, mais provient surtout des difficultés à faire la distribution éventuelle de tels excédents.

Dans cette situation, un groupe d'associations patronales dans la province de Québec, se sentant *affectées d'une manière discriminatoire par l'application du RRQ », ont présenté au Gouvernement Provincial des recommandations afin de corriger cette situation; d'ailleurs une semblable requête a été soumise précédemment par d'autres organismes au Gouvernement Fédéral, car - comme nous l'avons indiqué - les dispositions légales sont pratiquement les mêmes dans le RRQ que dans le RPC. Il ressort du mémoire québecois que, d'après une déclaration du Ministère fédéral du Revenu National, le * trop perçu • de la part des employeurs soumis à la RPC (donc dans les autres provinces que Québec) peut être estimé à $\$ 18$ millions par année; les associations québecoises estiment le «trop perçu " au Québec, sur une base proportionnelle, entre $\$ 7$ et $\$ 8$ millions par année, donc au total au Canada à environ $\$ 25$ millions par année.

Pour éviter une telle injustice grevant les employeurs, les pétitionnaires suggèrent * que les contributions du Régime soient identifiées au registre des cotisations du Régime " ce qui d'après le mémoire serait possible avec les ordinateurs électroniques à la disposition du Régime.

Mais même si une telle identification tait possible, avec des frais relativement modestes, la question se poserait à savoir à qui il faut 
rembourser l'excédent versé: faut-il le faire, par ordre chronologique, au premier employeur du salarié, ou bien au deuxième etc, ou encore proportionnellement à chacun d'eux, ou encore autrement. Il nous semble que la plus opportune serait la répartition proportionnelle à l'instar du remboursement aux salariés qui participent durant l'année aussi bien au RRQ qu'au RPC.

Pour notre part, nous pourrions suggérer une autre méthode afin d'éviter le * trop perçu * de la part des employeurs dans la plupart des cas, sans la nécessité d'identifier les contributions de ceux-ci.

Cette méthode consisterait dans la modification du principe pour le calcul des contributions des salariés (et par le fait même de leurs employeurs) recevant plus que le maximum des gains admissibles et cotisables; ce maximum devrait être fixé non seulement pour l'année totale, comme c'est le cas actuellement $(\$ 5,000$ pour les salaires admissibles et $\$ 4,400$ pour les salaires cotisables), mais en fonction de toute période de paie; par exemple pour la paie mensuelle: $\$ 416.67$ et $\$ 366.67$ respectivement; pour la paie à toutes les deux semaines: $\$ 192.30$ et $\$ 169.30$ respectivement, etc.

La contribution du salarié et de son employeur pour chaque période serait donc limitée au montant qui correspond à $1.8 \%$ du maximum du salaire cotisable pour la période en question, donc par exemple $\$ 6.60$ pour la paie mensuelle, $\$ 3.05$ pour la paie à toutes les deux semaines, etc.

A la méthode préconisée ici, on peut reprocher qu'elle pénalise les salariés qui, tout en gagnant durant l'année $\$ 5,000$ ou plus, ne travaillent qu'une partie de l'année, parce que leur salaire de l'année concernée pour le calcul des prestations, ne serait basé que sur les cotisations déduites à la source (y compris la part de l'employeur).

Pour éviter cette pénalisation, on n'a qu'à appliquer mutatis mutandis les dispositions de l'article 52 du RRQ (ou le paragraphe 3 de l'article 12 du RPC) qui permet, comme nous l'avons déjà mentionné, de considérer comme gain admissible du travail autonome, le montant par lequel son revenu durant l'année moins son exemption personnelle (avec maximum de $\$ 5,000$ ) excède le montant qui correspond aux contributions au taux de $3.6 \%$, c'est-à-dire comme travailleur autonome, sur la différence en question.

La méthode ici préconisée ne pourra pourtant remédier à la situation des salariés qui travaillent simultanément chez deux ou plusieurs employeurs, obtenant de chacun d'eux une rémunération moindre que le maximum des salaires admissibles. 